\title{
28 Research Suare \\ 18F-FDG-PET/CT Plays a Key Role in Formulating Treatment Strategies for Takayasu Arteritis
}

\section{Qing Han}

Xijing Hospital

Xiang Zhou

Xijing Hospital

jin ding

Xijing Hospital

zhaohui zheng

Xijing Hospital

Fei Kang

Xijing Hospital

Kui zhang

Xijing Hospital

Fengfan Yang

Xijing Hospital

Jinlin Miao

Xijing Hospital

jing wang

Xijing Hospital

Ping Zhu ( $\square$ zhupingfmmu@163.com )

Xijing Hospital

Original research

Keywords: Takayasu Arteritis, remission, 18F-FDG-PET/CT, inflammation

Posted Date: August 10th, 2020

DOl: https://doi.org/10.21203/rs.3.rs-55731/v1

License: (9) This work is licensed under a Creative Commons Attribution 4.0 International License.

Read Full License 


\section{Abstract}

Backgroud and Objective: Clinical evaluation alone cannot satisfy the prognosis of TAK. PET-CTmolecular imaging may be a useful tool to supplement the prognosis of TAK. The purpose of this study was to observe whether THE TAK patients who achieved clinical remission also achieved imaging remission with PET-CT. Pet-ct plays an important role in the prognosis of TAK patients and further formulation of treatment strategy.

Results: 79\% patients with TAK in remission were positive for ${ }^{18} \mathrm{~F}-\mathrm{FDG}-\mathrm{PET} / \mathrm{CT}$. The study population had a mean age of 38.8 years and was predominantly female (90\%). Their mean disease duration was 3.5 years and they had a mean disease remission period of 9.4 months. Patients had a mean ESR of 37.3 $\mathrm{mm} / \mathrm{h}$ and a mean CRP level of $13.5 \mathrm{mg} / \mathrm{l}$ at the first dignosis time and $4.9 \mathrm{~mm} / \mathrm{h} / 3.7 \mathrm{mg} / \mathrm{l}$ at the Courrent time point. There was a statistically significant difference between the two groups $(p<0.01)$. All patients on initial treatment received oral glucocorticoids, $25 \%$ received methotrexate, $65 \%$ received leflumide, and $45 \%$ received cyclophosphamide. Quantitative analysis of the SUV of nineteen patients showed an increasing trend (Baseline SUVmax $2.16 \pm 0.46$ vs Remission SUVmax 2.08 $\pm 0.49, p=0.56$; Baseline SUVmean $2.34 \pm 0.57$ vs Remission SUVmean $2.42 \pm 0.81, p=0.46$ ). The arterial SUVmax uptake was higher in 15 patients with remission than baseline (2.30 \pm 0.62 vs $2.48 \pm 0.91, p=0.54)$. But it was not statistically significant. Most of the patients $(13 / 19,68 \%)$ had an SUVmax value $\geq 2.0$ in visual vasculitis with positive PET/CT before treatment, and $32 \% \otimes 2.0$. No correlation was found between the type of treatment used, the time that elapsed from remission, or laboratory parameters and the scintigraphic results.

Conclusion: TAK's clinical remission criteria are not entirely consistent with actual vascular inflammatory activity. Patients with TAK that achieve clinical remission may not achieve imaging remission with PET$\mathrm{CT}$, and PET-CT plays an important role in the prognosis of TAK patients and further formulation of treatment strategy.

\section{Backgroud}

Takayasu Arteritis (TAK) is a Chronic, idiopathic, inflammatory disease that primary granulomatous large vessel vasculitis commonly affects the aorta and its immediate branches as well as pulmonary artery ${ }^{[1]}$. It is the key and difficult problem in TAK diagnosis and treatment to be able to accurately judge the activity of arteritis and timely detect the recurrence of the disease. The aim of treatment in TAK is directed at suppressing the inflammatory process and establishing a state of remission. Traditional disease modifying antirheumatic drugs (DMARDs) resulted in achieving clinical remission in $30-50 \%$ of patients in various clinical settings ${ }^{[2]}$. However, the definition of remission in TAK remains controversial. The preliminary criteria for disease activity proposed by Kerr GS et al. in 1994 that were adopted by the American College of Rheumatology (ACR) include four signs and symptoms of which two or more of the four must be satisfied were seemed as disease activity ${ }^{[3]}$. TAK is a rare disease with multiple nonspecific clinical symptoms. It is difficult to diagnose in the early stages of the disease. A variety of biomarkers are 
being developed to diagnose and evaluate their activity. Imaging techniques are required to monitor the disease course and the treatment response of the drug in TAK. Increased vascular wall thickness, vascular wall edema, and wall lining enhancement are generally considered to be evidence of disease activity ${ }^{[4]}$. Studies in east Asia have shown that aortic regurgitation, pulmonary hypertension, and elevated serum nt-pro-bnp levels in TAK patients are associated with disease activity ${ }^{[5]}$. Recent studies have shown that there is a discrepancy between the clinical and laboratory parameters that indicate a state of remission and between various imaging techniques that assess vascular inflammation ${ }^{[6]}$.. Erythrocyte sedimentation rate (ESR) and C-reactive protein (CRP) are commonly used to evaluate the activity of arteritis, but previous studies have shown that they are normal in active patients. However, ESR and CRP normal does not mean the regression of vascular wall inflammation and the cessation of lesions, and these two indicators can be elevated in many cases, which lacks specificity for the evaluation of vascular inflammation ${ }^{[7-9]}$. Conventional angiography,considered the 'gold standard' to assess vasculitis is invasive and associated with morbidity, radiation exposure and risk of contrast toxicity ${ }^{[10]}$. Since its usefulness in vasculitis was confirmed in $1999,{ }^{18} \mathrm{~F}$-FluoroDeoxyGlucose $\left({ }^{18} \mathrm{~F}-\mathrm{FDG}\right)$ positron emission tomography (PET) has gained widespread acceptance in the diagnosis and monitoring of TAK ${ }^{[11]}$. Studies have shown that the sensitivity and specificity of ${ }^{18} \mathrm{~F}$-FDG-PET/CT in the diagnosis of macrovasculitis are $92 \%$ and $100 \%$, respectively ${ }^{[12]}$.

Furthermore, ${ }^{18} \mathrm{~F}$-FDG-PET/CT can evaluate the efficacy of drug therapy of TAK ${ }^{[13,14]}$. Decreased vascular absorption in ${ }^{18} \mathrm{~F}$-FDG-PET/CT scan is associated with clinical remission. It should be noted that some patients after treatment with immunosuppressive agents, their clinical symptoms are relieved and the inflammatory markers are reduced to the normal range, but the ${ }^{18}$ F-FDG-PET/CT scan still shows abnormal carotid artery, suggesting that the lesion may still change to a low level of activity ${ }^{[15]}$.The proposed mechanism that stands behind the idea of using ${ }^{18} \mathrm{~F}$-FDG-PET/CT lies in the fact that In inflammatory tissues, glucose metabolism is increased, and the uptake of radiolabelled glucose can detect its activity ${ }^{[9]}$. Even in the case of steroid therapy, ${ }^{18} \mathrm{~F}-\mathrm{FDG}-\mathrm{PET} / \mathrm{CT}$ is possible to distinguish between active and inactive patients. This theory is further strengthened by the fact that similar results of biodistribution have been achieved by using ${ }^{18} \mathrm{~F}-\mathrm{FDG}-\mathrm{PET} / \mathrm{CT}^{[13]}$. Nevertheless, although nuclear medicine investigations have become more specific with the introduction of these new methods, their use is still limited due to cost and availability in various centers as compared to the use of ${ }^{18} \mathrm{~F}$-FDG-PET/CT.

In the present study we examined a cohort of TAK patients in clinical remission in order to find out whether clinical and laboratory parameters are consistent with the actual inflammatory activity in the vessel assessed by ${ }^{18}$ F-FDG-PET/CT.

\section{Patients And Methods}

\section{Patients}


Nineteen patients from a large cohort of TAK patients attending the rheumatology outpatient clinic of Xijing Hospital were randomly selected. Female(89\%), Age( $37.8 \pm 1.4 \mathrm{yrs}$, range15-40), Disease duration (3.5 $\pm 0.75 \mathrm{yrs}$, range2.6-5.1). Among the 19 patients in this study, Glucocorticoid(Glu) alone accounted for $5 \%$, and Glu + Methotrexate (MTX) + Leflunomide(LEF)for 20\%,Glu + Cyclophosphamide(CYS) + LEF for $25 \%$,Glu + CYS for $20 \%$,Glu + LEF + Tacrolimus (TAC) for $15 \%$,Glu + LEF for $5 \%$. Patients were included if they fulfilled the following criteria:1) TAK diagnosed according to the $\mathrm{NIH}$ criteria ${ }^{[16]}$; 2) Disease duration of more than 2 years; 3 ) No disease flares in the preceding 6 months period; 4) After the patient reached clinical remission, the treatment copy remained unchanged for at least 6 months. Exclusion criteria of the study were pregnancy, liver or renal function impairment, heart failure, respiratory failure, diabetes mellitus, psychological or neurological disorders and malignancy. All patients had to satisfy both the preliminary $\mathrm{NIH}$ criteria for remission. Clinical remission was defined as the absence of new or aggravated systemic and/or local symptoms and/or signs, blood pressure stabilization, ESR and CRP reduction to normal, and no new onset or progression of existing lesions on imaging.

\section{${ }^{18}$ F-FDG -PET/CT acquisition of image and image analysis}

All the patients received ${ }^{18} \mathrm{~F}$-FDG PET/CT scans on the same scanner (Biograph 40, Siemens) consistent with the standard clinical protocols ${ }^{[17,18]}$. ${ }^{18} \mathrm{~F}-\mathrm{FDG}$-PET/CT was performed using a PET/CT scanner (Biograph 40,SIEMENS) 60 minutes after intravenous injection of approximately $3.7 \mathrm{MBq}(0.1 \mathrm{mCi}) / \mathrm{kg}$ of ${ }^{18} \mathrm{~F}-\mathrm{FDG}$. The blood glucose levels that were checked before FDG administration were below $200 \mathrm{mg} / \mathrm{dl}$. PET data were reconstructed iteratively with and without attenuation correction based on CT data and reoriented in axial, sagittal, and coronal slices. Objective reference values were obtained for all patients by measuring mean standard uptake values (SUV mean) of mediastinal blood pool and liver. Vascular-wall FDG uptakes were quantified by drawing volume of interests (irregular and voxel) in the ascending aorta, arcus aorta, descending aorta, abdominal aorta, right brachiocephalic artery, right subclavian artery, left subclavian artery, right common carotid artery, left common carotid artery, and pulmonary arteries were not included due to their close relationship with histological intense cardiac FDG uptake. The quantified ${ }^{18} \mathrm{~F}-\mathrm{FDG}$ uptake using methods as standard uptake value (SUV). The mean SUV in the center of the inferior vena cava in all cases, and target/background ratio was calculated as max SUV in arterial wall/mean SUV in inferior vena cava in their study ${ }^{[19]}$. A max SUV cut-off was set at 2.0 for detecting active inflammation of TAK in untreated cases and a cut-off for max SUV (strong accumulation, SUV > 2.0; weak accumulation, SUV 1.2-2.0 ${ }^{[20]}$. 
Table 1

Clinical and laboratory data of the study group.

\begin{tabular}{|c|c|c|c|}
\hline \multirow[t]{2}{*}{ Variables } & \multicolumn{2}{|l|}{ Study group } & \multirow[t]{2}{*}{ p-value } \\
\hline & Baseline & Remission & \\
\hline $\mathrm{M} / \mathrm{F}$ ratio & $2 / 17$ & $2 / 17$ & NS \\
\hline Age (yrs.) & $37.8 \pm 1.4$ & $37.8 \pm 1.4$ & NS \\
\hline (range) & $15-40$ & $15-40$ & NS \\
\hline Disease duration (yrs.) & $3.5 \pm 0.75$ & $3.5 \pm 0.75$ & NS \\
\hline (range) & $2.6-5.1$ & $2.6-5.1$ & NS \\
\hline Disease remission period (months) & NA & $9.35 \pm 1.71$ & NS \\
\hline (range) & NA & $7-12$ & NA \\
\hline $\operatorname{ESR}(\mathrm{mm} / \mathrm{h})^{a}$ & $37.3 \pm 28.77$ & $4.89 \pm 2.67$ & 0.004 \\
\hline CRP (mg/liter) ${ }^{b}$ & $13.52 \pm 5.62$ & $3.72 \pm 2.46$ & 0.007 \\
\hline \multicolumn{4}{|l|}{ Medication therapy } \\
\hline Glu alone & $0 / 20(0 \%)$ & $1 / 20(5 \%)$ & NS \\
\hline Glu + MTX + LEF & $5 / 20(25 \%)$ & $4 / 20(20 \%)$ & NS \\
\hline Glu + CYS + LEF & $4 / 20(20 \%)$ & $5 / 20(25 \%)$ & NS \\
\hline Glu + CYS & $4 / 20(20 \%)$ & $4 / 20(20 \%)$ & NS \\
\hline Glu + LEF + TAC & $3 / 20(15 \%)$ & $3 / 20(15 \%)$ & NS \\
\hline Glu + LEF & $2 / 20(10 \%)$ & $1 / 20(5 \%)$ & NS \\
\hline \multicolumn{4}{|c|}{$\begin{array}{l}\text { Glu:Glucocorticoid;MTX:methotrexate;LEF:leflunomide;CYS:cyclophosphamide.NS: non } \\
\text { significant.Values are presented as mean } \pm \text { standard deviation. Erythrocyte sedimentation rate (ESR) } \\
\text { and C-reactive protein (CRP). }{ }^{\text {a }} \text { ESR lower detection limit is } 20 / 15\left(\text { Fmale/Male) } \mathrm{mm} / \mathrm{h} \text {; }{ }^{\mathrm{b}} \mathrm{CRP} \text { lower }\right. \\
\text { detection limit is } 8 \mathrm{mg} / \mathrm{L} .\end{array}$} \\
\hline
\end{tabular}

\section{Results}

Demographic and clinical features A total of 19 TAK patients who satisfied the preliminary NIH criteria for remission were studied. The demographic and clinical features of the study group are presented in Table 1. The study population had a mean age of 38.8 years and was predominantly female $(90 \%)$. Their mean disease duration was 3.5 years and they had a mean disease remission period of 9.4 months. Patients had a mean ESR of $37.3 \mathrm{~mm} / \mathrm{h}$ and a mean CRP level of $13.5 \mathrm{mg} / \mathrm{l}$ at the first dignosis time and $4.9 \mathrm{~mm} / \mathrm{h} / 3.7 \mathrm{mg} / \mathrm{l}$ at the current time point. There was a statistically significant difference between the two groups $(p<0.01)$. All patients on initial treatment received oral glucocorticoids, $25 \%$ received 
methotrexate, $65 \%$ received leflumide, and $45 \%$ received cyclophosphamide. Most patients received a combination of glucocorticoids and DMARDs.

\section{${ }^{18}$ F-FDG -PET/CT}

In the overall cohort of our TAK patients, Nineteen patients in remission (19/19, 100\%) were positive for $\mathrm{PET} / \mathrm{CT}$. The most common PET/CT findings were found in Aorta abdominalis (68\%) and Ascending aorta (68\%) followed by Aaortic arch (63\%), Arteria carotis communis (53\%), Arteria Subclavia (47\%) and Renal artery (21\%). The pulmonary artery, Brachial Artery, Trunk brachiocephalic artery and Iliac artery were involved in $11 \%$ of patients. Twenty-one percent of TAK patients achieved imaging remission during remission. The diseased arteries improved from baseline in remission included: Aorta abdominalis, Aaortic arch, Renal artery and Arteria iliaca communis. There was no statistically significant difference between baseline and remission for all arteries involved ( $p \otimes 0.5)$. Quantitative analysis of the SUV of 19

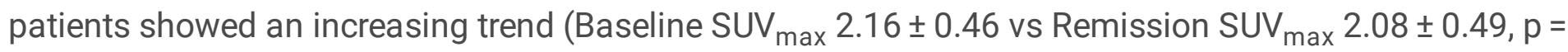
0.56; Baseline SUV mean $2.34 \pm 0.57$ vs Remission SUV mean $2.42 \pm 0.81, p=0.46$ ), Only $21 \%$ achieved imaging remission with PET/CT. The arterial SUV $\mathrm{Vax}_{\text {max }}$ uptake was higher in 15 patients with remission than baseline ( $2.30 \pm 0.62$ vs $2.48 \pm 0.91, p=0.54)$. But it was not statistically significant Fig. 1 . Most of the patients $(13 / 19,68 \%)$ had an SUVmax value $\geq 2.0$ in visual vasculitis with positive PET/CT before treatment, and $32 \% \otimes 2.0$. Figure 2 shows PET/CT SUV changes between remission and baseline in pulmonary artery. The values of pulmonary artery SUV $\mathrm{max}_{\text {ax }}$ in the remission stage (1.94) were lower than that in the baseline stage (2.65), and the values in the baseline stage (SUV mean 1.96$)$ were in the disease activity stage, while the values in the remission stage $\left(S U V_{\text {mean }} 2.02\right)$ were in the non-disease activity stage, but there was no statistical difference.

\section{Discussion}

TAK is a chronic, idiopathic, inflammatory disease that primarily affects the great vessels ${ }^{[21]}$. Due to its non-specific clinical manifestations, lack of specific biomarkers, characteristic imaging changes, and lack of a unified treatment regimen. Therefore, there are still some difficulties in the diagnosis and treatment of TAK. Especially, it is difficult to diagnose, assess the activity, evaluate the curative effect and evaluate the prognosis of atypical arteritis. In a subset of patients with signs and symptoms of active disease, serum reactant levels did not increase during the acute phase.In other patients who appear to have no disease, there is laboratory or radiological evidence ${ }^{[16]}$. Although the NIH criteria all use inflammatory markers such as ESR and CRP as part of the assessment, the methods used to assess disease activity or remission status are not sufficient and sensitive enough to detect low levels of vascular inflammation. ${ }^{18}$ F-FDG PET/CT combines the functional information of PET with the anatomical information of $\mathrm{CT}^{[22]}$.This is a new diagnostic tool that can show vascular inflammation before any structural changes. ${ }^{18}$ F-FDG PET/CT is a non-invasive imaging method that measures the accumulation of ${ }^{18} \mathrm{~F}$-FDG in the highly metabolized and activated inflammatory cell infiltration vessels ${ }^{[23]}$. In inflammatory tissue, glucose metabolism is increased, and its activity can be measured by the uptake of radio-labeled glucose ${ }^{[24]}$. Even 
when treated with steroids, it is possible to distinguish between active and inactive patients ${ }^{[24]}$. In our study, TAK patients also met NIH remission criteria by using PET/CT technology, while active subclinical vascular inflammation was still present. The PET/CT method we used is only functional and only related to the activity of vascular inflammation. Similar to the studies of Grayson et al., PET/CT can predict the recurrence and mitigate the risk ${ }^{[25]}$.

In addition, we found no association between the type of drugs used by patients (including hormones, anti-tnf therapy, and immunosuppressants) and the outcome of the inflammatory process. However, due to the limited number of reported cases, the safety and long-term efficacy of these drugs in TAK-CAD remains to be further investigated. The Ishikawa and Maetani study showed that among 120 TA patients, the 15 -year survival rate was $93 \%$ for those with prolonged remission, the 15-year survival rate for people with persistent active disease was 68 percent. Those with both persistent and severe vascular disease had a 15-year survival rate of just 40 percent. The reported mortality rate of patients with ta-cad was between $3 \%$ and $21.0 \%[26]$. These large differences are due to different study methods, different follow-up times, and different demographic/clinical and therapeutic differences. However, our study makes us realize that some limitations may lead us to bias in interpreting the data. The number of patients in remission in this study was too small, so it is necessary to further expand the sample size and continue to observe the prognosis of such patients. Whether patients and doctors should continue treatment after TAK clinical remission and accurately assess patients' disease activity is a challenging clinical issue at present. It is of great significance to find appropriate clinical assessment methods and guide clinical treatment to prolong patients' survival and reduce mortality.Our results suggest that vascular inflammation and injury in TAK patients in clinical remission is still progressing, providing evidence for further enhancement of treatment. Therefore, angiography alone seems to be inadequate as a means of assessing TAK structural damage. Other models, such as power doppler ultrasound, MRI, and PET/CT, should be used to comprehensively assess disease activity. These results may also prompt researchers to modify the current criteria for TAK mitigation to meet modern standards, including clinical, laboratory and imaging parameters.

\section{Conclusion}

TAK's clinical remission criteria are not entirely consistent with actual vascular inflammatory activity. Patients with TAK that achieve clinical remission may not achieve imaging remission with PET-CT, and PET-CT plays an important role in the prognosis of TAK patients and further formulation of treatment strategy.

\section{Abbreviations}

Takayasu Arteritis (TAK), Traditional disease modifying antirheumatic drugs(DMARDs),American College of Rheumatology (ACR),Erythrocyte sedimentation rate (ESR) ,C-reactive protein (CRP), ${ }^{18} \mathrm{~F}$ - 
FluoroDeoxyGlucose $\left({ }^{18} \mathrm{~F}-\mathrm{FDG}\right)$ positron emission tomography (PET), mean standard uptake values (SUV) ,Glucocorticoid (Glu),Methotrexate (MTX),Cyclophosphamide(CYS), Tacrolimus (TAC) .

\section{Declarations}

Acknowledgements:Thanks to all authors for their contributions and participation.Funding:The authors state that this work has not received any funding. Compliance with Ethical Standards.Guarantor:The scientific guarantor of this publication is Professor Ping Zhu. Conflict of Interest:The authors of this manuscript declare no relationships with any companies. Statistics and Biometry:No complex statistical methods were necessary for this paper. Informed Consent:Only if the study is on human subjects:Written informed consent was obtained from all subjects (patients) in this study. Ethical Approval:Institutional Review Board approval was obtained.No study subjects or cohorts overlap.

9. Methodology:observational,multicenter study.

Acknowledgements:

Thanks to all authors for their contributions and participation.Funding:The authors state that this work has not received any funding. Compliance with Ethical Standards.Guarantor:The scientific guarantor of this publication is Professor Ping Zhu. Conflict of Interest:The authors of this manuscript declare no relationships with any companies. Statistics and Biometry:No complex statistical methods were necessary for this paper. Informed Consent:Only if the study is on human subjects:Written informed consent was obtained from all subjects (patients) in this study. Ethical Approval:Institutional Review Board approval was obtained.No study subjects or cohorts overlap. 9.

Methodology:observational,multicenter study.

\section{References}

1. Kim E, Beckman J. Takayasu arteritis: challenges in diagnosis and management[J]. Heart. 2018;104(7):558-65.

2. Sesin CA, Bingham CR. Remission in rheumatoid arthritis: wishful thinking or clinical reality?[J]. Semin Arthritis Rheum. 2005;35(3):185-96.

3. Kerr GS, Hallahan CW, Giordano J, et al. Takayasu arteritis[J]. Ann Intern Med. 1994;120(11):919-29.

4. Tombetti E, Mason JC. Application of imaging techniques for Takayasu arteritis[J]. Presse Med. 2017;46(7-8 Pt 2):e215-23.

5. Kong X, Ma L, Wu L, et al. Evaluation of clinical measurements and development of new diagnostic criteria for Takayasu arteritis in a Chinese population[J]. Clin Exp Rheumatol. 2015;33(2 Suppl 89):48-55.

6. Hoffman GS, Ahmed AE. Surrogate markers of disease activity in patients with Takayasu arteritis. A preliminary report from The International Network for the Study of the Systemic Vasculitides (INSSYS)[J]. Int J Cardiol. 1998;66(Suppl 1):191-4, S195. 
7. Holley L, Woodland N, Hung WT, et al. Influence of fibrinogen and haematocrit on erythrocyte sedimentation kinetics[J]. Biorheology. 1999;36(4):287-97.

8. Dhingra R, Gona $\mathrm{P}, \mathrm{Nam} \mathrm{BH}$, et al. C-reactive protein, inflammatory conditions, and cardiovascular disease risk[J]. Am J Med. 2007;120(12):1054-62.

9. Sato El, Lima DN, Espirito SB, et al. Takayasu arteritis. Treatment and prognosis in a university center in Brazil[J]. Int J Cardiol. 2000;75(Suppl 1):163-6.

10. Webb M, Al-Nahhas A. Molecular imaging of Takayasu's arteritis and other large-vessel vasculitis with 18F-FDG PET[J]. Nucl Med Commun. 2006;27(7):547-9.

11. Blockmans D, Knockaert D, Maes A, et al. Clinical value of [(18)F]fluoro-deoxyglucose positron emission tomography for patients with fever of unknown origin[J]. Clin Infect Dis. 2001;32(2):191-6.

12. Tezuka D, Haraguchi G, Ishihara T, et al. Role of FDG PET-CT in Takayasu arteritis: sensitive detection of recurrences[J]. JACC Cardiovasc Imaging. 2012;5(4):422-9.

13. Iwabu M, Yamamoto Y, Dobashi H, et al. F-18 FDG PET findings of Takayasu arteritis before and after immunosuppressive therapy[J]. Clin Nucl Med. 2008;33(12):872-3.

14. de Leeuw K, Bijl M, Jager PL. Additional value of positron emission tomography in diagnosis and follow-up of patients with large vessel vasculitides[J]. Clin Exp Rheumatol. 2004;22(6 Suppl 36):216.

15. Andrews J, Al-Nahhas A, Pennell DJ, et al. Non-invasive imaging in the diagnosis and management of Takayasu's arteritis[J]. Ann Rheum Dis. 2004;63(8):995-1000.

16. Kerr GS, Hallahan CW, Giordano J, et al. Takayasu arteritis[J]. Ann Intern Med. 1994;120(11):919-29.

17. Slart R. FDG-PET/CT. (A) imaging in large vessel vasculitis and polymyalgia rheumatica: joint procedural recommendation of the EANM, SNMMI, and the PET Interest Group (PIG), and endorsed by the ASNC[J]. Eur J Nucl Med Mol Imaging. 2018;45(7):1250-69.

18. Dejaco C, Ramiro S, Duftner C, et al. EULAR recommendations for the use of imaging in large vessel vasculitis in clinical practice[J]. Ann Rheum Dis. 2018;77(5):636-43.

19. Tezuka D, Haraguchi G, Ishihara T, et al. Role of FDG PET-CT in Takayasu arteritis: sensitive detection of recurrences[J]. JACC Cardiovasc Imaging. 2012;5(4):422-9.

20. Kobayashi Y, Ishii K, Oda K, et al. Aortic wall inflammation due to Takayasu arteritis imaged with 18FFDG PET coregistered with enhanced CT[J]. J Nucl Med. 2005;46(6):917-22.

21. Keser G, Aksu K, Direskeneli H. Takayasu arteritis: an update[J]. Turk J Med Sci. 2018;48(4):681-97.

22. Kim J, Oh MD. FDG PET-CT in the Diagnosis of Takayasu Arteritis Presenting as Fever of Unknown Origin: A Case Report[J]. Infect Chemother. 2015;47(3):190-3.

23. Alexanderson-Rosas E, Monroy-Gonzalez AG, Juarez-Orozco LE, et al. [(18)F]-Sodium fluoride uptake in Takayasu arteritis[J]. J Nucl Cardiol. 2017;24(5):1674-9.

24. Ratanapo S, Srivali N, Ungprasert P, et al. Role of FDG-PET/CT in diagnosis of aortitis caused by Takayasu arteritis and IgG4-related systemic disease[J]. Heart. 2013;99(24):1876. 
25. Grayson PC, Alehashemi S, Bagheri AA, et al. (18) F-Fluorodeoxyglucose-Positron Emission

Tomography As an Imaging Biomarker in a Prospective, Longitudinal Cohort of Patients With Large Vessel Vasculitis[J]. Arthritis Rheumatol, 2018,70(3):439-449.

26. Ha KJ, Cho WC, Kim WK, et al. A Minimally Invasive Approach for the Treatment of Mid-Aortic Syndrome in Takayasu Arteritis[J]. Korean J Thorac Cardiovasc Surg. 2018;51(6):399-402.

\section{Tables}

"Due to technical limitations, table 2 is only available as a download in the Supplemental Files section.

\section{Figures}

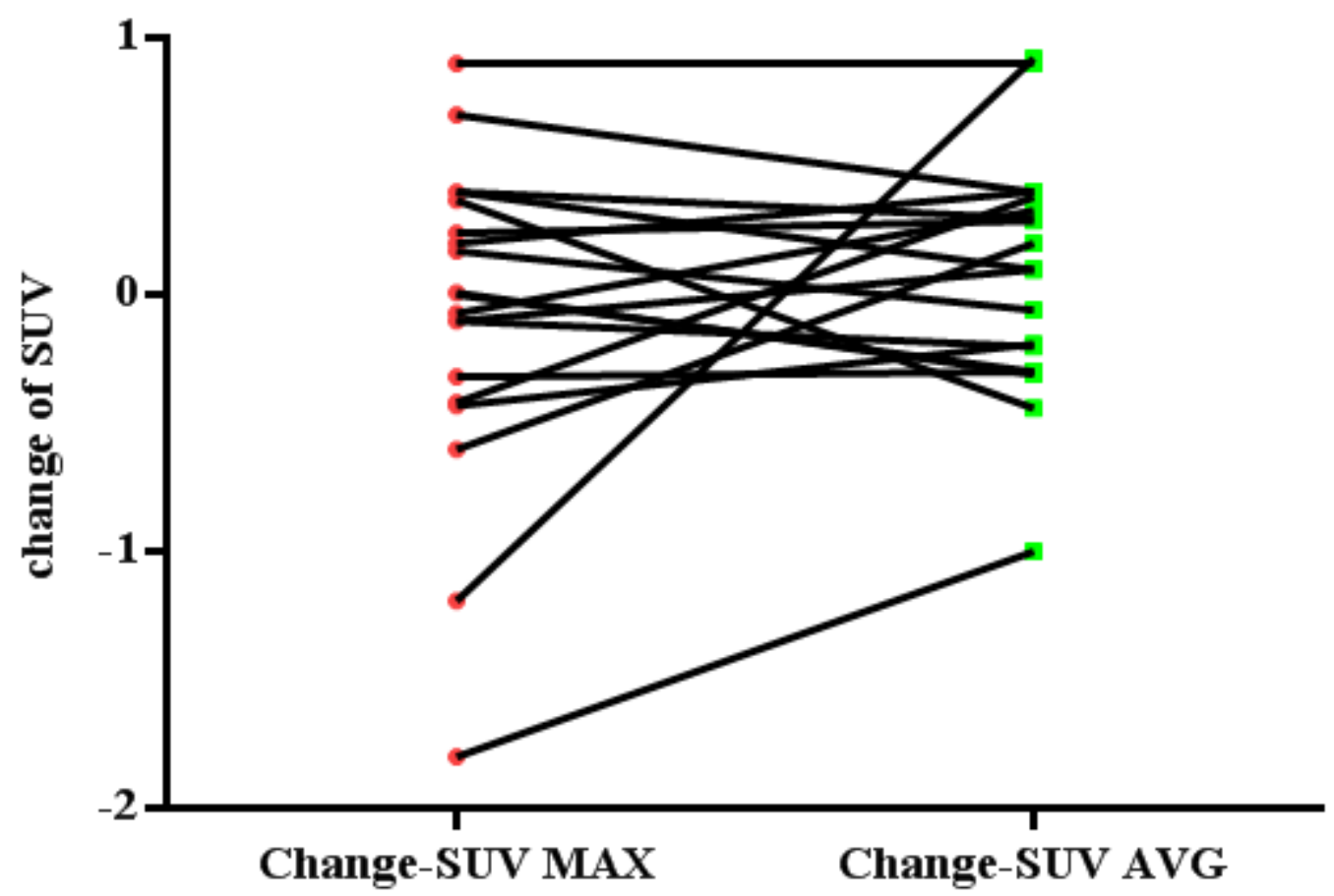

Figure 1

shows the changes in SUVmax $(-0.09 \pm 0.63)$ and SUVmean $(-0.08 \pm 0.46)$ between baseline and remission in TAK patients. There was no statistical significance in the remission stage of nineteen TAK patients compared with the baseline stage. Only 4 TAK patients were in non-disease activity in remission stage, and $79 \%$ TAK patients were still in imaging disease activity in remission stage. 

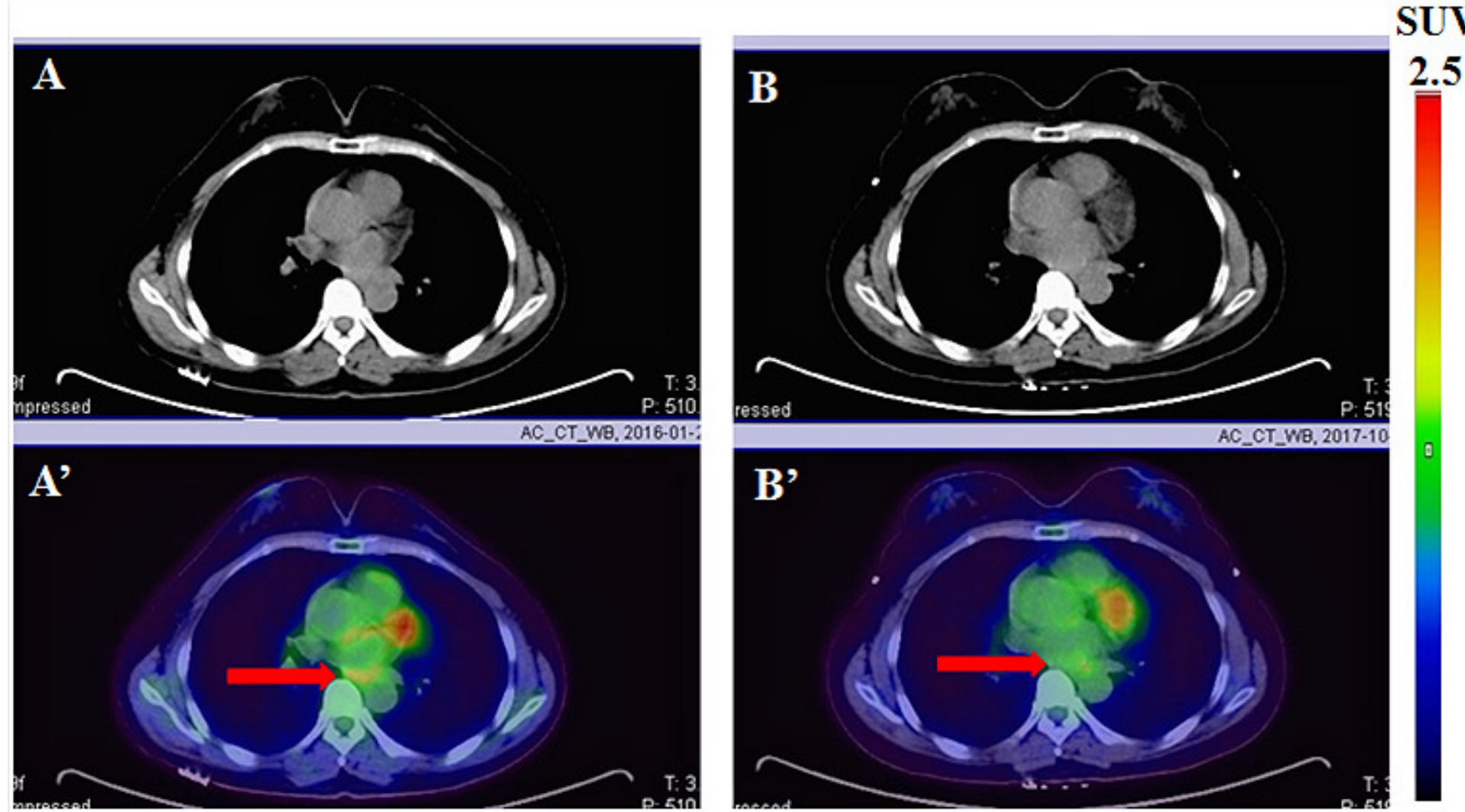

Figure 2

Example of a 47 positron emission tomography (PET) image of the Pulmonary artery of a patient with TAK who had multiple PET-positive vascular inflammation. SUVmax in baseline (January 2016) was 2.65 QSUVmean 1.96冈, and in remission (October 2017) was 1.94囚SUVmean 2.02】. Red arrow represents one PET-positive joint (green/yellow). SUV standardized uptake values.

\section{Supplementary Files}

This is a list of supplementary files associated with this preprint. Click to download.

- table1.jpg 